\title{
Coupled cell networks: Boolean perspective
}

\author{
Katarzyna (Kasia) Świrydowicz \\ Department of Mathematics \\ Virginia Polytechnic Institute \\ Blacksburg, VA, USA \\ kswirydo@vt.edu
}

Received: 13 March 2015, accepted: 22 March 2017, published: 9 May 2017

\begin{abstract}
In this paper we use Boolean framework to redefine coupled cell networkss, originally described in [2]. We also analyze some of the important properties of Boolean coupled cell networkss.

In the second part of this paper we focus on properties of a quotient networks. We redefine the concept of a quotient to suit Boolean network framework. Next, we investigate in details the networks in which two-cell bidirectional ring and three-cell bidirectional ring arise as quotients.
\end{abstract}

Keywords-Boolean networks; coupled cell networks; discrete models

\section{INTRODUCTION}

During the 1980s and early 1990s, Martin Golubitsky and Ian Stewart formulated and developed a theory of coupled cell networkss (CCNs) [2]. Their research was primarily focused on quadrupeds' gaits. Since they were particularly interested in the change of synchrony between four legs of an animal, they needed a special framework to describe this phenomenon. For example, they were interested in how does the synchrony of four legs change when the animal speeds up from walk to gallop.

The most important concept in the CCN theory is a cell. The cell captures the dynamics of one unit of the system (for example, one leg of an animal) and the dynamical system consists of many identical cells connected to each other. Each cell has its own state space and evolution equation(s). Even though models based on identical cooperating units are common in many areas especially in biology, ecology and sociology, [4], [5], [6], [7], [8] - the CCN setup helps to formulate questions in terms of symmetry and synchrony rather than system evolution as a whole.

In this paper, we redefine coupled cell networkss using the framework of Boolean networks [9], [10]. This moves the theory to a new setting. As expected, some phenomena turns out to be very similar as for continuous networks and some others do not. In addition we study the phenomena specific to Boolean networks and not arising in continuos dynamical systems.

We note that the Boolean coupled cell networkss are a subclass of Boolean networks, which differs both from the original Kaufman's Boolean switching nets [9], and from cellular automata [23], [24]. In his work [9], Kaufman focused on networks with topology based on $k$-regular graph, which makes the topology similar to that of CCNs, however, he chose update rules for each of the nodes randomly. In contrast, in this paper we assume that the update rules for each cell-node in the network are identical. In Cellular Automata the update rules are the same for every cell-node in the network, and this makes Cellular Automata 
similar to Boolean CCNs. The difference is that a state of a cell in a cellular automaton depends only on the states of its immediate neighbors, and this formulation does not allow for any irregularity in the network structure. A cell in a cellular automaton might not depend on the cells that are far away from its physical location on the grid. In contrast, such a dependence can occur in Boolean CCNs. Hence, Boolean CCNs share some characteristics with Boolean switching nets and cellular automata, and in fact, can be treated as a cross between these two species of Boolean networks.

The main result in this paper is the analysis of the Boolean CCNs in which two cell bidirectional ring and three-cell bidirectional ring arise as a quotient.

This paper is organized as follows. Section II contains the definitions of the concepts needed to describe a Boolean network, and it also containts the definition of coupled cell networks. Section III describes the main problem we want to address in this paper. Section IV points out the differences between continuous and Boolean dynamics. In the Section $\mathrm{V}$ we define the quotient network, and form the rules for taking quotients. In this section we study our first example case, the networks for which two cell bidirectional ring arise as a quotient. Section VII provides a biological model in which the ideas from the previous sections are used. Section $\mathrm{VI}$ is devoted to analyzing the networks for which three-cell bidirectional ring arise as a quotient. In the Section IX we present conclusions and ideas for the future.

\section{PREliminaries}

\section{A. Boolean functions and dynamical systems}

The definitions contained in this subsection come from the classic literature on Boolean functions and networks, see [9], [10], [12], [15]

By Boolean function we understand a function $f: \mathbb{F}_{2}^{n} \rightarrow \mathbb{F}_{2}$. Let $x_{i} \in \mathbb{F}_{2}, i=1, \ldots, n$. The Boolean function can be represented in the form

$$
f\left(x_{1}, x_{2}, \ldots, x_{n}\right) .
$$

A Boolean dynamical system is a set of $n$ ordered Boolean functions from $\mathbb{F}_{2}^{n}$ to $\mathbb{F}_{2}$. First function is an update function for the first variable, second function is an update function for the second variable, and so on. Thus, a Boolean dynamical system is defined as $F: \mathbb{F}_{2}^{n} \rightarrow \mathbb{F}_{2}^{n}$ where

$$
\begin{aligned}
F & =\left(f_{1}, f_{2}, \ldots, f_{n}\right) \\
& =F\left(f_{1}\left(x_{1}, x_{2}, \ldots, x_{n}\right), \ldots, f_{n}\left(x_{1}, x_{2}, \ldots, x_{n}\right)\right)
\end{aligned}
$$

There is some ambiguity in the literature on how multiplication and addition are defined in the Boolean algebra. For example, Francis Robert [10] defines $1 * 1=0$, whereas other authors (for example [18]) consider $1 * 1$ to be 1 . Except of adding and multiplying variables, we are also allowed to add 1 , which is equivalent to negation. Throughout this paper we use the multiplication and addition tables given in Figure 1 .

\begin{tabular}{c|c||cr|c||c}
$\mathrm{x}$ & $\mathrm{y}$ & $\mathrm{x}+\mathrm{y}$ & $\mathrm{x}$ & $\mathrm{y}$ & $\mathrm{x}$ * \\
\hline \hline 1 & 1 & 0 & 1 & 1 & 1 \\
1 & 0 & 1 & 1 & 0 & 0 \\
0 & 1 & 1 & 0 & 1 & 0 \\
0 & 0 & 0 & 0 & 0 & 0 \\
\multicolumn{5}{c}{$\mathrm{x}$} & $\mathrm{x}+1$ \\
\multicolumn{5}{c}{1} & 0 \\
& 0 & 1
\end{tabular}

Fig. 1. Multiplication and addition in $\mathbb{F}_{2}$

Multiplication can be also expressed with the logical operator AND $(\wedge)$. If $X$ and $Y$ are Boolean variables, then $X$ AND $Y=1$ if and only if the value of both $X$ and $Y$ is 1 (the logical value is true). The truth table is then identical to the one for multiplication.

The addition operation is equivalent to $\mathrm{XOR}$ $(\underline{\vee}) . X$ XOR $Y$ is true only when either $X$ is true or $Y$ is true, and false when both are true or both are false.

Negation $(\neg)$ is equivalent to adding 1 to variable. $X+1=0$ if $X=1$ and $X+1=1$ if $X=0$. By adding 1 we flip the value of the variable.

Hence, the alternative formulation with AND, XOR and NEG is

A Boolean dynamical system is a discrete time system. For a system of a size $n$ there are $2^{n}$ 
Katarzyna Świrydowicz, Coupled cell networks: Boolean perspective

\begin{tabular}{|c|c|c|c|c|c|}
\hline $\mathrm{X}$ & $\mathrm{y}$ & x XOR y & $\mathrm{x}$ & $\mathrm{y}$ & $\mathrm{x}$ AND y \\
\hline$\overline{c 1}$ & $\overline{1} 1$ & 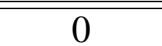 & $\bar{~} 1$ & $\bar{~} 1$ & $\overline{c 1}$ \\
\hline 1 & 0 & 1 & 1 & 0 & 0 \\
\hline 0 & 1 & 1 & 0 & 1 & 0 \\
\hline 0 & 0 & 0 & 0 & 0 & 0 \\
\hline & & $\mathrm{x}$ & \multicolumn{3}{|c|}{ NEG $x$} \\
\hline & & 1 & \multicolumn{3}{|l|}{0} \\
\hline & & 0 & \multicolumn{3}{|l|}{1} \\
\hline
\end{tabular}

Fig. 2. Multiplication and addition in $\mathbb{F}_{2}$

possible states of a system. Throughout this paper we will assume that all variables are updated simultaneously.

While classifying Boolean dynamical systems, we are interested in two phenomena. The first one is the occurrence and the number of steady states (attractors). The steady state (SS) of a Boolean system is a state $\left(x_{1}, x_{2}, \ldots, x_{n}\right)$ which updates to itself, i.e. $F\left(x_{1}, x_{2}, \ldots, x_{n}\right)=\left(x_{1}, x_{2}, \ldots, x_{n}\right)$.

The second phenomenon is the number and length of cycles. Let $\left(x_{1}, x_{2}, \ldots, x_{n}\right)$ be a starting state. If after $p>1$ updates the system returns to the starting state, we say that a system has a cycle of length $p-1$.

Unlike for continuous systems, we do not have the tools coming from bifurcation theory. Most methods are based on statistics, algebra, combinatorics and topology, see [9], [12], [13], [14]. We notice that a Boolean dynamical system must either have a steady state or a cycle or both; there is no possibility of oscillations and chaotic behaviors, which occur for continuous networks.

\section{B. Coupled cell networkss: idea}

The idea of a coupled cell networks is to look at the dynamical system not as a whole, but rather to look at the dynamics of particular members of the system. In order to do so, we divide the system into separate entities called cells. The cell captures one one or more differential equations. The dynamics of the cell depends upon the cell itself (self-variable(s)) and couplings (variables of other cells). There may be more than one type of coupling since cells may interact with each other in many different ways.
We can easily represent a coupled cell networks as a graph. The vertices of the graph are the cells, and couplings are the edges of the graph. The different types of couplings are shown as different types of edges.

\section{Boolean coupled cell networks: formal defini- tions}

Definition 1. By cell we understand an entity of the n-dimensional Boolean dynamical system together with its update function.

Definition 2. By coupling we understand an influence that one cell has on the dynamics of the other cell.

In this paper, we will consider only regular networks. The cells of regular network are all identical and there is only one type of coupling. We assume that every cell has the same number of couplings (this is enforced by the property of all cells being identical). Every cell has only one self-variable.

We assume that if some number of cells couple to cell $A$, then we can permute the variables of coupling cells and we get the same equation up to permutation of variables. The last statement comes from the assumption that there is only one type of coupling. The statement can be formalized as

$$
x_{k}=f\left(x_{k}, \overline{x_{k 1}, \ldots, x_{k m}}\right)
$$

where $x_{k 1}, \ldots, x_{k m}$ are the variables of coupling cells, and overline indicates that we can permute them. By convention, we write the self-variable in the first position. Here $f$ stands for a function template. Since every cell is governed by the same equation, the template is the same, however, since cells have different couplings (but always the same number of couplings) the functions are not identical.

Thus, the regular Boolean coupled cell networks is represented as 


$$
\begin{aligned}
& x_{1}=f\left(x_{1}, \overline{x_{11}, \ldots, x_{1 m}}\right), \\
& x_{2}=f\left(x_{2}, \overline{x_{21}, \ldots, x_{2 m}}\right), \\
& \ldots \ldots \ldots . . \\
& x_{n}=f\left(x_{n}, \overline{x_{n 1}, \ldots, x_{n m}}\right) .
\end{aligned}
$$

The network shown above has valency $m$, which means that every cell receives inputs from $m$ other cells.

In this paper we consider only update functions whose formula depends on the variable of self and all the variables of the couplings. For example, in a valency 1 network a function

$$
f\left(x_{1}, x_{2}\right)=x_{1}+x_{2}
$$

is a valid function, whereas

$$
f\left(x_{1}, x_{2}\right)=x_{2}
$$

is not a valid function. We call the valid functions admissible functions.

In order to fully define Boolean coupled cell networks we need a function template and a graph of connections.

Example 1. Consider function scheme $F(x, y)=$ $x+x * y$ and a graph of connection (further called architecture graph) shown in Figure 3

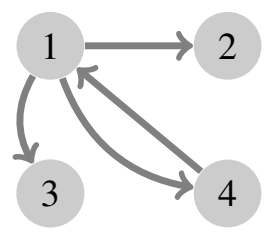

Fig. 3. Example 1: a graph of connections in a regular Boolean CCN

Using the template and the graph, we obtain

$$
\begin{aligned}
& x_{1}=x_{1}+x_{1} * x_{4}, \\
& x_{2}=x_{2}+x_{2} * x_{1}, \\
& x_{3}=x_{3}+x_{3} * x_{1}, \\
& x_{4}=x_{4}+x_{4} * x_{1} .
\end{aligned}
$$

Lemma 1. The number of function templates that could be used in a Boolean CCN with $n$ cells and valency $m<n$ is given as $2^{2 m+2}$

Proof: We consider the transition table associated to the Boolean CCN. Every cell in this network is influenced by $m$ other cells. Hence, the template function for this network depends on $m+1$ variables (variable of self and $m$ variables of couplings). For a given cell, the function is $f\left(x, y_{1}, \ldots, y_{m}\right)$.

To fully describe a Boolean function on $m+1$ variables, we need to create a transition table and assign a 0 or a 1 to all possible $2^{m+1}$ states. This gives 2 choices for every of $2^{m+1}$ places, which is in total $2^{2^{m+1}}$ possibilities.

As we stated before, couplings are insensitive to permutation. For a given cell, let us set up the variable of self to be 1 . Then, we assign 0 or 1 to a state where all couplings are 0 s, then 0 or 1 to a state when one coupling is 1 (we emphasize that it does not matter which of the couplings is 1 ), two couplings are 1 s, and so on until we reach the state where all the couplings are 1 . In total, we have a choice in $2^{m+1}$ places.

Next, we set up the variable of self to be 0 and we repeat the same process. We get $2^{m+1} \cdot 2^{m+1}$ possibilities. We have then $2^{2 m+2}$ possible function templates for a network with valency $m$.

\section{Problem Statement}

Dynamical systems arising in biology and ecology are often large [16]. Large networks are hard to analyze mathematically, both from discrete and from continuous point of view [15], [17]. Usually in such cases a model reduction technique is applied [13], [17], [18], [19]. The authors of [1] base their model reduction strategy intended for CCNs on cell coloring. They cluster cells with the same color. This clustered network is called a quotient network, which is formally defined in Section V. In addition to defining the rules for forming a quotient network, the authors of [1] go further. They look at the quotient network and ask what are the networks that admits this quotient, 
and, if we know the properties of the quotient, what can be concluded about the original network?

In the next sections we perform a similar analysis for Boolean CCNs, and we demonstrate analogous results. We give some insights about the influence of the network architecture on the network dynamics.

\section{NETWORK DYNAMICS}

A continuous dynamical system is often defined with one or more parameters [20]. We do not have this advantage for Boolean dynamical systems and enforcing the use of parameters is somewhat artificial. This issue is discussed in details in [11] and references therein. Bifurcations are tied to parameters; there are no bifurcations in the Boolean dynamical systems.

The authors of [1] focused on synchronybreaking (pitchfork) bifurcations that are common in coupled cell networkss and in some cases, quotient is able to predict their existence in the original network.

For Boolean CCNs, instead of looking for bifurcations, we look for steady states and cycles. We show that a small Boolean CCN (with 2, 3 and 4 cells) could not have both cycles and steady states in the same network.

We define a few concepts related to the dynamics of a Boolean CCN.

By canonical steady state we understand a state of a system when all the cells are working at the same way. We have 2 such states for a Boolean network: $(0,0, \ldots, 0)$ and $(1,1, \ldots, 1)$.

An interesting phenomenon that happens in Boolean coupled cell networkss is that once synchronized, the network could not un-synchronize, because all the cells use the same update function. In all Boolean coupled cell networks we have either canonical steady states or canonical cycle (a cycle when the system alternates between two canonical states).

Canonical Steady States and Canonical Cycles are called the canonical part of the dynamics.

In addition, Boolean coupled cell networkss often have non-canonical parts, which are steady states and cycles where the system is not synchronized. The appearance of such structures depends on the functions and on the architecture graph.

\section{QUOTIENTS NETWORKS}

All the results shown in [1] regarding quotients apply directly to Boolean systems, because these results are based on graph theory and combinatorics but not on the network dynamics.

Hence, we just re-state the principles of coloring and taking quotients defined in [1].

By coloring we understand the function that assigns a color to every cell (node). Of course one graph could be colored in many different ways.

By balanced coloring we understand a coloring for which every cell with color $a$ receives the same number of inputs from the cells with color $b$, for each $b$. An example of balanced coloring is shown in Figure 4.

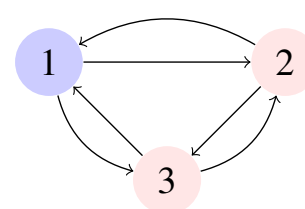

Fig. 4. An example of balanced coloring in $\mathrm{CCN}$.

A quotient is defined based on coloring. All cells with the same color become one meta-cell. The result is shown in Figure 5 .

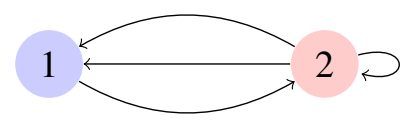

Fig. 5. An quotient network for the network from Figure 4 formed based on coloring.

Taking a quotient affects the functions associated with the cells. This means that all the variables of the cells clustered to one meta-cell are replaced by one variable.

In the examples shown in Figure 4 and Figure 5. the original system of three equations 


$$
\begin{aligned}
& x_{1}=f\left(x_{1}, \overline{x_{2}, x_{3}}\right), \\
& x_{2}=f\left(x_{2}, \overline{x_{1}, x_{3}}\right), \\
& x_{3}=f\left(x_{3}, \overline{x_{1}, x_{3}}\right),
\end{aligned}
$$

changes to

$$
\begin{aligned}
& x_{1}=f\left(x_{1}, \overline{x_{2}, x_{2}}\right), \\
& x_{2}=f\left(x_{2}, \overline{x_{1}, x_{2}}\right),
\end{aligned}
$$

\section{NETWORKS THAT ADMITTWO-CELL} BIDIRECTIONAL RING AS A QUOTIENT: CASE
STUDY

One of the example cases considered in [1] is a network named two cell bidirectional ring (shown in Figure 6).

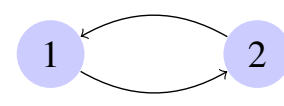

Fig. 6. two cell bidirectional ring

Note. The network presented in Figure 6 should not be confused with the diagram of mutual activation/inhibition that often appears in mathematical biology papers [25]. The function that describes mutual activation/inhibition is a function that assigns the cell the state of its coupling. This is not an admissible function in the context of CCNs, since it does not involve cell's own state variable in the update formula.

A circuit in a graph is a path consisting of vertices and edges with the property that we can reach a vertex from itself. We note that networks that admit two-cell bidirectional ring as a quotient have a structure of a bipartite graph with in-degree 1. Such a graph could have only one circuit, and if it had two, it would be disjoint) Hence the graph that admits the two-cell bidirectional ring is a circuit with some attached structure, influenced by the dynamics of the circuit, but not influencing back. We will call all the graphs having this structure $G_{2 C B R}$. An example of such a structure is shown in Figure 7.

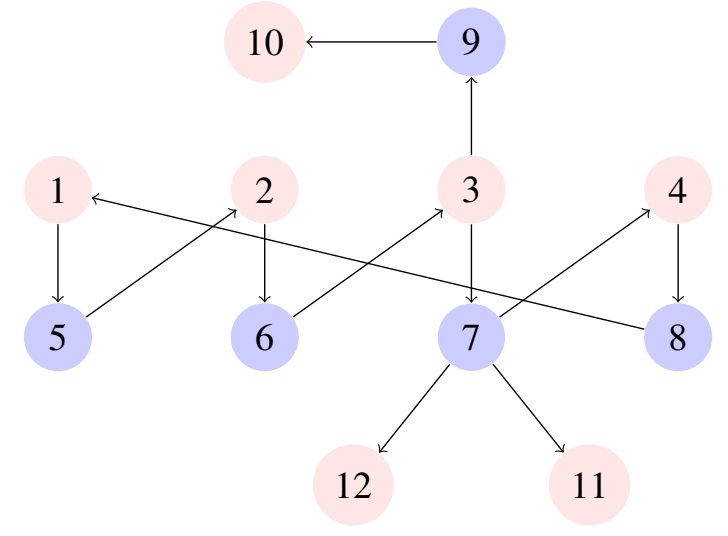

Fig. 7. An example pf $G_{2 C B R}$ graph

Claim. The dynamics of the system built on the $G_{2 C B R}$ architecture depends on the dynamics of its single circuit. In particular, if there is a steady state in the circuit, there is a steady state in the system. If there is a cycle of length 2 or more, there is a cycle in the entire system.

Proof: Let us observe that the structure of all $G_{2 C B R}$ graphs is a circuit plus some attached structure. We will look at the attached part. The cells belonging to the circuit are influenced only by other cells that belong to the circuit. If a cell belongs to the attached structure, it must receive input from either a cell from the circuit or from another cell that does not belong to the circuit. If the attached structure is non-empty, there is at least one cell in the attached structure that receives input from the cell from the circuit, because the graph is connected.

Assume that the circuit achieves a steady state. Then, all the cells directly influenced by the circuit achieve steady state as well. The same happens with the cells influenced by these cells.

Assume that the circuit achieves a cycle of length greater than 1 . This means that the circuit oscillates between two or more states. The inputs received by non-circuit cells are either changing or stay steady. In any case, since the non-circuit part does not influence the circuit part, the entire 
system could not go to a steady state and there must be a cycle for the entire system.

Lemma 2. Let $G$ be a graph from the $G_{2 B C R}$ family. Let $F(a, b)$ be a two-variable Boolean update function template, where $a$ is the variable of self and $b$ is the variable of a coupling cell.

The network $X=\{G, F\}$ could either have a non-canonical cycle(s) or non-canonical steady state(s), but never both.

Proof: Let us first use the observation from [1] that the adjacency matrix of such an architecture must have the structure (proved in [1])

$$
\left[\begin{array}{cc}
C & 0 \\
B_{1} & B_{2}
\end{array}\right]
$$

where $C$ is the matrix of a circuit and $B_{2}$ is a lower triangular matrix with 0 s on the diagonal. We can imagine such architecture as a circuit of length $l$ with attached non-circuit structure.

We investigate the dynamics of the circuit alone. Based on the previous claim, the dynamics of the non-circuit part strictly depends on the dynamics of the circuit.

Let us assume that there are $l$ cells in the circuit. We will write a state of the circuit as $\left(s_{1}, s_{2}, \ldots, s_{l}\right)$ assuming that $s_{1}$ sends input to $s_{2}$, $s_{2}$ to $s_{3}$ and so forth and $s_{l}$ sends input to $s_{1}$.

Let us assume that we have a non-canonical steady state in this structure. This means that this steady state of the form $(\ldots, 0,1,0, \ldots)$ or $(\ldots, 1,0,1, \ldots)$

In both cases we have $F(1,0)=1$ and $F(0,1)=0$.

Note that we cannot have both $F(0,0)=1$ and $F(1,1)=0$, since this leads to a function $F(a, b)=b+1$ (which is not admissible). We could not have $F(1,1)=1$ and $F(0,0)=0$ because this leads to $F(a, b)=a$, which is not admissible as well.

We have two cases:

- Case 1: $F(0,0)=0$ and $F(1,1)=0$. We notice that a system driven by such a function could not oscillate. Once changed to 0 , a cell could not go back to 1 .
- Case 2: $F(0,0)=1$ and $F(1,1)=1$. We notice that here oscillations are impossible as well. Once a state of a variable is changed to 1 , it could not go back to 0 .

We conclude that if Boolean $\mathrm{CCN}$ from the $G_{2 B C R}$ family has a non-canonical steady state, it cannot have a non-canonical cycle.

To prove the converse, let us assume that there is a non-canonical cycle in $\{G, F\}$.

As a part of this cycle we must have a transition between two states of the system as shown below

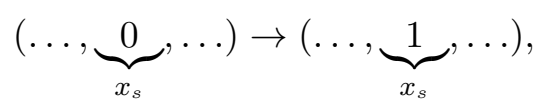

or

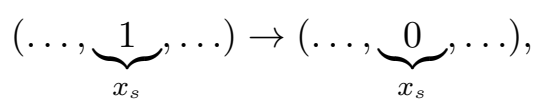

Thus, for the first case we must have $F(0,0)=$ 1 or $F(0,1)=1$ and for the second case $F(1,0)=$ 0 or $F(1,1)=0$.

- Case $1, F(0,0)=1$. There are 4 possible options (note that either $F(1,0)=1$ or $F(1,1)=1$ because $x_{s}$ must eventually return to the original state.

1)

$$
\begin{aligned}
& F(0,1)=0 \\
& F(1,0)=0 \\
& F(1,1)=1
\end{aligned}
$$

In this case $F(a, b)=a+b+1$. If there exists a non-canonical steady state, we must have for some $x_{k}$ and $x_{k+1}$

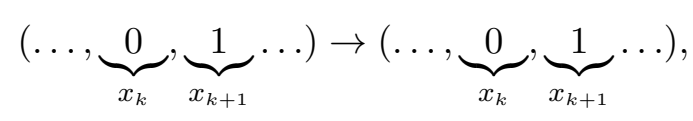

This is, however, impossible because $F(1,0)=0$.

2)

$$
\begin{aligned}
& F(0,1)=0 \\
& F(1,0)=1 \\
& F(1,1)=0
\end{aligned}
$$

In this case we have $F(a, b)=1+b$ and this is not a valid update function 
(it does depend only on the coupling variable).

3)

$$
\begin{aligned}
& F(0,1)=1 \\
& F(1,0)=0 \\
& F(1,1)=1
\end{aligned}
$$

By the same reasoning as in 1) we obtain that this function could not produce a non-canonical steady state.

4)

$$
\begin{aligned}
& F(0,1)=1 \\
& F(1,0)=1 \\
& F(1,1)=0
\end{aligned}
$$

By the same reasoning as in 1) and in 3), we obtain that this function could not produce a non-canonical steady state.

- Case $1, F(0,1)=1$

Based on similar reasoning as in the previous case, points 1), 3) and 4), the template function with such a property could not produce a non-canonical steady state.

The proof for Case 2 is analogous. Thus, we obtain that if there is a non-canonical cycle in $\{G, X\}$, then there cannot be a non-canonical steady state.

We conclude that non-canonical steady states and non-canonical cycles do not appear together in a Boolean $\mathrm{CCN}$ that admits two-cell bidirectional ring as a quotient.

Next, we use the observation that the dynamics of the non-circuit part of the system depends on the circuit. Hence, if there is no oscillation in the circuit, there are no oscillations in the entire system.

Theorem 3. The following are true:

1) If the 2CBR has non-canonical steady states, so does the non-quotient network, from which it arose.

2) If the 2CBR has non-canonical cycles, so does the non-quotient network, from which it arose.

\section{Proof:}

There are only $2^{2 \cdot 1+2}=16$ Boolean coupled cell networks that could be created on a $2 \mathrm{CBR}$ architecture. Eight of them yield only canonical dynamics and eight do not. We need to exclude all the networks where we do not have both the influence of self-variable and of the coupling. Eventually we are left with four networks.

These are the systems that have non-canonical cycles:

$$
\begin{aligned}
& f_{1}=x_{1} * x_{2}+x_{1}+1 \\
& f_{2}=x_{2} * x_{1}+x_{2}+1
\end{aligned}
$$

and

$$
\begin{aligned}
& f_{1}=x_{1} * x_{2}+x_{2}, \\
& f_{2}=x_{2} * x_{1}+x_{1} .
\end{aligned}
$$

These are the systems that have non-canonical steady states.

$$
\begin{aligned}
& f_{1}=x_{1} * x_{2}+x_{2}+1 \\
& f_{2}=x_{2} * x_{1}+x_{1}+1
\end{aligned}
$$

and

$$
\begin{aligned}
& f_{1}=x_{1} * x_{2}+x_{1}, \\
& f_{2}=x_{2} * x_{1}+x_{2} .
\end{aligned}
$$

Again, we can use the structure that admits 2CBR as a quotient. We know that this structure consists of a circuit and some circuit-dependent cells that do not form a circuit themselves.

Similarly as in the proof of Lemma 2, we can just consider the dynamics of the circuit.

We analyze the above-mentioned four systems separately.

The first system gives $F(0,0)=1, F(1,1)=1$, $F(0,1)=1$ and $F(1,0)=0$. We can assume that the cells influence each other in an order (the first cell influences the second, the second influences the third and so on, the $n$th cell influences the first cell) and consider any starting state, say 
$(1,0,0, \ldots, 0)$. From the dynamics we have a sequence of states

$$
\begin{gathered}
(1,0,0, \ldots, 0) \\
(0,1,1, \ldots, 1), \\
(1,0,1, \ldots, 1), \\
(1,1,0, \ldots, 1), \\
\ldots \\
(0,1,1, \ldots, 1)
\end{gathered}
$$

which is clearly a cycle of length 2 or more. Since the rest of the dynamics is influenced by the dynamics of the cells belonging to the circuit and we have "pulses" of 0 s and $1 \mathrm{~s}$, we can only end up having a cycle for the entire structure.

By Lemma 2, cycles and steady states do not appear simultaneously and we have a system with additional cycles.

The second case leads to $F(0,0)=0, F(1,1)=$ $0, F(0,1)=1, F(1,0)=0$. If we start with $(1,0, \ldots, 0)$, we obtain

$$
\begin{gathered}
(1,0,0, \ldots, 0), \\
(0,1,0, \ldots, 0), \\
(0,0,1, \ldots, 0), \\
\ldots \\
(0,0,0, \ldots, 1), \\
(1,0,0, \ldots, 0),
\end{gathered}
$$

which is a cycle of length 2 or more. By the same line of reasoning as for the first case, we get a system with additional cycles.

The third case gives $F(0,0)=1, F(1,1)=$ $1, F(0,1)=0$ and $F(1,0)=1$. Let us use the same argument as for the first case and consider the circuit separately, and assume that it is ordered. Let us take a starting state, say $(1,0, \ldots, 0)$. Based on $F$, we obtain a sequence of system states

$$
\begin{gathered}
(1,0,0, \ldots, 0), \\
(1,0,1, \ldots, 1), \\
(1,0,1, \ldots, 1), \\
\ldots \\
(1,0,1, \ldots, 1),
\end{gathered}
$$

and $(1,0,1, \ldots, 1)$ is clearly a non-canonical steady state.

Because of the lack of the circuit in the rest of the system architecture, we must have a steady state for the entire system. By the lemma, we must have a system with additional steady states.

The fourth case gives us $F(0,0)=0, F(1,1)=$ $0, F(0,1)=0$ and $F(1,0)=1$. We start with $(1,0, \ldots, 0)$. We obtain a sequence of system states

$$
\begin{gathered}
(1,0,0, \ldots, 0), \\
(1,0,0, \ldots, 0), \\
\ldots \\
(1,0,0, \ldots, 0),
\end{gathered}
$$

and $(1,0,0, \ldots, 0)$ is clearly a non-canonical steady state. By similar reasoning as in previous cases and Lemma 2 we obtain that this system must have non-canonical steady states.

\section{BIOLOGICAL EXAMPLE}

Most known Boolean models in systems biology are characterized by cooperating species, each of which is governed by a different set of rules [18], [26]. Models based on the idea of identical entities governed by identical sets of rules are quite common in ecology, however these models are usually not Boolean [27].

To illustrate a Boolean network that admits twocell bidirectional ring as a quotient, we use a simple fish schooling model. A reaction of a fish school to a predator is a well-documented behavior [29]. There exist a couple of theories explaining this phenomenon. One of the theories is a many eyes hypothesis [28]. According to this theory, the advantage of swimming in a schools is that the fish can rely on collective vigilance while avoiding predators, and thus spend more time foraging. Once a single fish senses a predator, it sends a signal to neighboring fish [30] (for example, changes the direction, and the neighboring fish are able to sense this change rapidly). These neighboring fish send signal further, until the entire school is 
alert and invokes its defense mechanisms (for example, collectively changes direction). Previously, fish schools have been modeled by both, an ODE model [31], and discrete agent-based model [27].

In very simplistic terms, a fish in a school has two possible states: alert and non-alert. An alert fish spreads the alert signal to other fish, causing them to change their state to alert. We can assume that a fish might send a signal to multiple fish, but receives a signal only from one other fish (say, closest lateral neighbor). In order for a school to ensure an efficient spread of information, we must have a cycle inside the fish network.

We assume that an alert fish stays alert regardless of the state of its coupling fish. In a school we also have fish who stay "inside" the school and base their safety on the vigilance of the more specialized fish. We note that there might exist some fishes, who are undervigilant and do not inform the surroundings, but are still able to receive the alert information.

Hence, in our network the nodes are fish, state 1 means that the fish is alert and state 0 means that it is not. A fish has only one coupling, but might be a coupling to many other fish. A simple illustration of such behavior is shown on the Figure 8 .

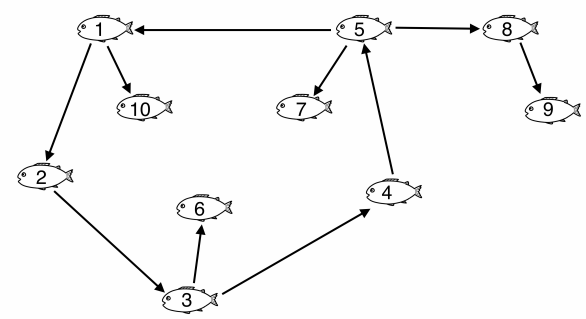

Fig. 8. Simple fish school model.

We note that in such a Boolean network, any state where any of the fish-nodes on the circuit is alert leads to a state where all the fishes are alert. Also a state where none of the fish is alert, is a steady state. We note that such dynamics is governed by a function $\mathrm{F}(0,1)=1$ (non-alert fish changes a state to alert once a coupling is alert), $F(1,0)=1$, and $F(1,1)=1$ (alert fish stays alert regardless if its coupling is alert or not)

\begin{tabular}{c|cccccccccc} 
Time step & 1 & 2 & 3 & 4 & 5 & 6 & 7 & 8 & 9 & 10 \\
\hline 1 & 0 & 1 & 0 & 0 & 0 & 0 & 0 & 0 & 0 & 0 \\
2 & 0 & 1 & 1 & 0 & 0 & 0 & 0 & 0 & 0 & 0 \\
3 & 0 & 1 & 1 & 1 & 0 & 1 & 0 & 0 & 0 & 0 \\
4 & 0 & 1 & 1 & 1 & 1 & 1 & 0 & 0 & 0 & 0 \\
5 & 1 & 1 & 1 & 1 & 1 & 1 & 1 & 1 & 0 & 0 \\
6 & 1 & 1 & 1 & 1 & 1 & 1 & 1 & 1 & 1 & 1
\end{tabular}

Fig. 9. Dynamics of fish school model with initial condition: fish 2 senses predator (time step 1).

and $F(0,0)=0$ (non-alert fish stays non-alert when the coupling is non-alert). In such a network there are canonical steady states $(0,0, \ldots, 0)$ and $(1,1, \ldots, 1)$, many possible non-canonical steady states, and no cycles. For example, in the network shown in Figure 8, all the states listed in the table below are the non-canonical steady states, and this is not a complete list

\begin{tabular}{|l|}
\hline$(0,0,0,0,0,1,0,0,0,0)$ \\
$(0,0,0,0,0,0,1,0,0,0)$ \\
$(0,0,0,0,0,0,0,1,0,0)$ \\
$(0,0,0,0,0,0,0,0,0,1)$ \\
$(0,0,0,0,0,1,1,0,0,0)$ \\
$(0,0,0,0,0,1,0,1,0,0)$ \\
$(0,0,0,0,0,1,0,0,0,1)$ \\
$(0,0,0,0,0,0,1,1,0,0)$ \\
$(0,0,0,0,0,0,1,0,0,1)$ \\
$(0,0,0,0,0,1,0,1,0,1)$ \\
$(0,0,0,0,0,1,0,0,1,1)$ \\
\hline
\end{tabular}

Fig. 10. Example of non-canonical steady states of the Boolean $\mathrm{CCN}$ shown in Figure 8

The network presented above reduces to a $2 \mathrm{CBR}$ with 2 steady states $(0,0)$ and $(1,1)$ and dynamics $(0,1) \rightarrow(1,1),(0,1) \rightarrow(1,1),(0,0) \rightarrow(0,0)$, $(1,1) \rightarrow(1,1)$. The function that governs this system is $F(x 1, x 2)=(x 1+1) *(x 2+1)+1=$ $\neg(\neg x 1 \wedge \neg x 2)$.

This shows that regardless of the size of the school, one can interpret its behavior in the same way: if one fish on the circuit is alert (i.e. fish who is specialized in vigilance), all fish become alert, and if none of the fish on the circuit is alert, the school stays non-alert. We note that if the fish 
that is not on the circuit becomes alert, it does not have an ability to inform the other fish. This can be thought of as either the fish is currently foraging, and the other, more specialized fish in the school would notice the predator anyways, or the fish is too far away from the school, or the fish just received false signal. The quotient network is an simplification of this system where only the dynamics of the cycle is taken into account.

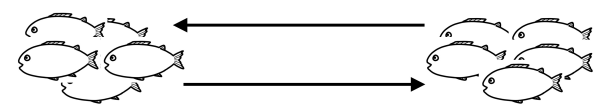

Fig. 11. Quotient of simple fish school model.

The model presented above is very simple, however, it illustrates the idea well. A behavior where one member of the species informs other members about the danger does occur in plants as well; for example tomato plants are able to spread such information [32].

\section{NETWORKS THAT ADMIT THREE-CELL BIDIRECTIONAL RING AS A QUOTIENT: CASE STUDY}

According to [1], there are two networks with 4 cells that admit three-cell bidirectional ring as a quotient and 12 networks with 5 cells that admit three-cell bidirectional ring as a quotient. The authors of [1] have shown that the dynamics of a three-cell bidirectional ring is a good predictor of the dynamics of a bigger network for both networks with 4 cells and for the 10 out of 12 networks with 5 cells.

The three-cell bidirectional ring is a structure shown in Figure 12 .

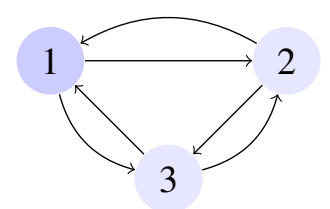

Fig. 12. three-cell bidirectional ring

The two networks with four cells admitting three-cell bidirectional ring as a quotient are shown in Figure 13, All twelve five-cell networks
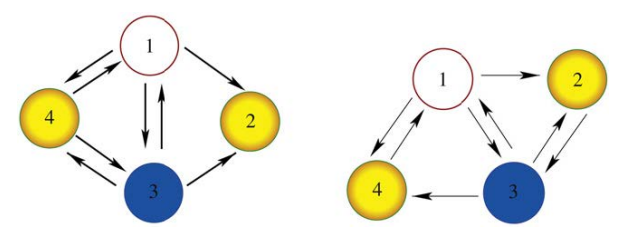

Fig. 13. Four-cell networks that admit three-cell bidirectional ring as a quotient, taken from [1]

admitting three-cell bidirectional ring as a quotient are shown in Figure 14. The analysis of the net-
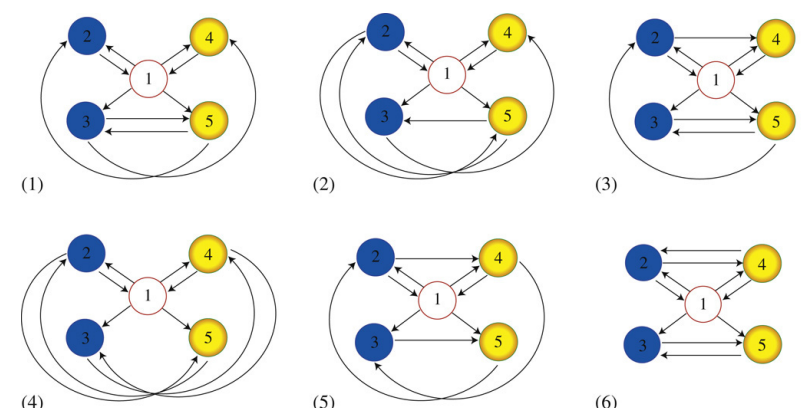

(4)
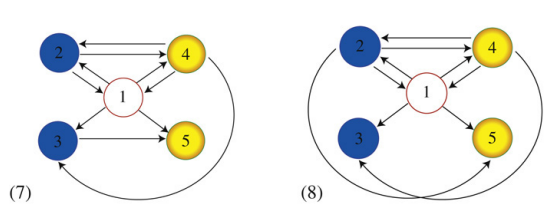

(6)
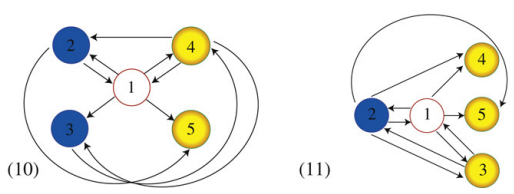

(9)
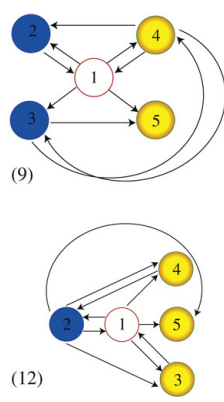

Fig. 14. Five-cell networks that admit three-cell bidirectional ring as a quotient, taken from [1]

works that admit a particular network as a quotient was based on the topology, not on the dynamics, hence we can use the results from [1].

There are 64 Boolean networks of valency 2 (regular network with two couplings). If we apply those networks to the three-cell bidirectional ring, it turns out that 32 of these networks have only canonical structure, and only 4 have additional cycles and the rest have additional steady states. One of the 4 networks is not admissible since the function template can be written as $F(a, \overline{b, c})=1+a$, 
hence only 3 remain. Similarly to the $2 \mathrm{CBR}$ case, we cannot have non-canonical steady states and non-canonical cycles in the same network using the three-cell bidirectional ring as an architecture graph.

Theorem 4. If a Boolean coupled cell networks of valency 2 that admits three-cell bidirectional ring as a quotient is built using any of the three function templates that result in additional cycles for the three-cell bidirectional ring, it could not have non-canonical steady states.

Proof:

- Network 1: For this network we have

$$
\begin{aligned}
& F(1,1,1)=0, \\
& F(1,1,0)=0, \\
& F(1,0,1)=0, \\
& F(1,0,0)=0, \\
& F(0,1,1)=1, \\
& F(0,0,1)=1, \\
& F(0,1,1)=1, \\
& F(0,0,0)=0,
\end{aligned}
$$

We notice that for this network once the cell changes its state to 1 , after update it changes to 0 regardless of what is the state of the coupling. Hence the only possible steady state is a canonical state with all 0 s.

- Network 2:

$$
\begin{aligned}
& F(1,1,1)=1, \\
& F(1,1,0)=0, \\
& F(1,0,1)=0, \\
& F(1,0,0)=0, \\
& F(0,1,1)=1, \\
& F(0,0,1)=1, \\
& F(0,1,1)=1, \\
& F(0,0,0)=1,
\end{aligned}
$$

We use the same line of reasoning and notice that once the cell has a state 0 , it must change the state to 1 regardless what is the state of couplings. The only steady state is such as a system could be a canonical state with all $1 \mathrm{~s}$.

- Network 3:

$$
\begin{aligned}
& F(1,1,1)=1, \\
& F(1,1,0)=0, \\
& F(1,0,1)=0, \\
& F(1,0,0)=0, \\
& F(0,1,1)=1, \\
& F(0,0,1)=1, \\
& F(0,1,1)=1, \\
& F(0,0,0)=0,
\end{aligned}
$$

Here the situation is not as clear as for the two previous functions.

Assume that we have a non-canonical steady state in this network. Since it is noncanonical, it must have some number of $0 \mathrm{~s}$ and $1 \mathrm{~s}$. If it is $(1,0,0, \ldots, 0)$, the first 1 is influenced by 20 s and we end up in a canonical steady state. The same happens for a state with 2 ones. If we take a state with $(1,1,1,0,0, \ldots, 0)$ and assume that first 3 cells influence each other and all the cells with 0 s influence each other we conclude that it is a steady state, but in such a case the network is disjoint. We use the same way of reasoning for all the states with 4 or more 1 s. We conclude that a network with such an update scheme could not have non-canonical steady states.

The characterization of the networks with noncanonical steady states is much harder. Depending on the architecture, the networks that admit three-cell bidirectional ring may have or may not have both non-canonical steady states and noncanonical cycles.

Claim The following is true for the networks that admit three-cell bidirectional ring as a quotient. 
- The dynamics of $\mathrm{s}$ three-cell bidirectional ring is a good predictor of a dynamics of the network with 4 cells that has admitted it. If a three-cell bidirectional ring has only noncanonical cycles, so does the bigger network. If a three-cell bidirectional ring has only additional steady states, so does the bigger network.

- For the 4 out of 125 -cell networks that admit a three-cell bidirectional ring as a quotient the dynamics of a three-cell bidirectional ring is a good predictor of the dynamics of a bigger network. For the rest of the networks, noncanonical cycles may appear, even though the smaller network has only non-canonical steady states.

A contribution towards the proof. As mentioned earlier, there exist 64 function templates for Boolean $\mathrm{CCN}$ created based on a three-cell bidirectional ring architecture. 32 of them have non-canonical dynamics. 28 functions out of the 32 have non-canonical steady states. We used a CPP code to test two 4-cell networks and twelve 5 -cell networks using each of the 28 functions.

- If a 3CBR quotient has a non-canonical steady state, so does the 4-cell network that has admitted it. If 3CBR has a non-canonical cycle, so does the 4-cell network that admitted it. This result was obtained using exhaustive computer simulation.

- For 4 graphs $(8,9,11$, and 12$)$, if the quotient network has only non-canonical steady states, so does the networks that admitted it. This result was obtained using exhaustive computer simulation.

- If the quotient network has only cycles, so does the network that admitted it. This is true for all 12 of the 5-cell networks. This result was obtained using exhaustive computer simulation.

Figure 15 shows how many (out of 28) functions cause the non-qoutient graph to have both noncanonical cycles and non-canonical steady states.

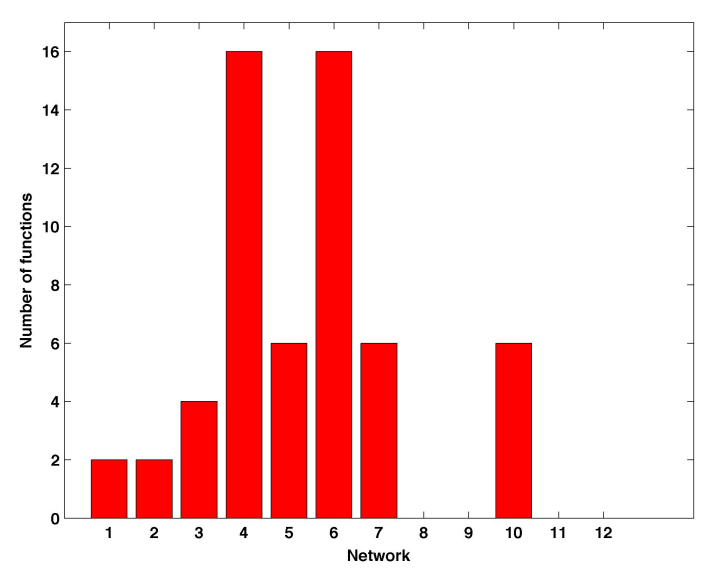

Fig. 15. Number of function templates that have have both non-canonical steady states and non-canonical cycles in a 5cell non-quotient network.

\section{Relationship with Agent-Based MODELS}

Every coupled cell networks can be viewed as an agent-based model. The agent-based model is a model consisting of identical interacting agents. The framework of agent-based models is very general; any object could be considered an agent (network cells, fishes, people susceptible to infection, bugs, trees etc). Every agent is described by the state vector (for example, position, infection, age, alertness) and the state vector is updated according to the same rules.

The model presented in Section VII can be viewed as agent-based model. In this model the fish function as agents. These fish are characterized by a state vector with only one variable: state of alertness (alert or non-alert). In this simple model fish are stationary, which means they do not move throughout the domain. The update rules for the state of a single agent are the same as described in Section VII.

Typically, agent-based models are large. There are not many mathematical methods that allow us to control (predict and change) their behavior [21], [22]. A method that allows a researcher to look at the quotient of the model and predict the behavior of the bigger original model would be a 
desirable tool. There exist a framework that makes it possible to translate an agent-based model into a system of Boolean equations [3], and thus, at least in some cases, it can be translated to a Boolean $\mathrm{CCN}$.

\section{CONCLUSIONS AND FUTURE WORK}

In this paper we show preliminary work regarding Boolean coupled cell networks. We are able to fully characterize the dynamics of the networks that admit two-cell bidirectional ring as a quotient, and we provide a small contribution to characterizing networks that admit three-cell bidirectional ring as a quotient. The main contribution in this paper is showing that CCNs have their Boolean counterpart and that this new setting brings a new perspective on Boolean functions and Boolean networks.

The phenomenon of bifurcations does not arise in Boolean CNNs, yet it does not make investigating their dynamics easy. In fact, predicting dynamics based on the quotient turns out to be a hard problem for the Boolean CCNs. In the future we plan to investigate bigger networks (with more that 5 cells) that bring $3 \mathrm{CBR}$ as a quotient, possibly using high performance computing. An efficient algorithm for easy enumeration and generation of such networks is needed as well.

In this paper we do not analyze the dependence between internal symmetries of the network graph and properties of its dynamics. Such analysis is an important part of CNN research [2] and we plan to develop similar techniques and ideas for Boolean CCNs.

Finding a more powerful, biology-related application of Boolean quotient networks is another goal. This would allow us to confirm the importance of the results derived in this paper on a new level.

In this paper we focus on regular Boolean CCNs with one type of coupling. Expanding the research to networks with less regularity and two or more types of coupling is another future goal.

\section{Acknowledgements}

This research was originally inspired by Dr. Reinhard Laubenbacher from the Center for Quantitative Medicine, Uconn Health, University of Connecticut, CT, USA.

The suggestions and comments of two anonymous reviewers on the earlier versions of this manuscript helped to improve the paper and make it more clear and readable.

\section{REFERENCES}

[1] D. Aguiar, A.P.S. Dias, M. Golubitsky, M. Leite, Bifuracations from Regular Quotient Networks: A First Insight., Physica D, 238 (2009) 137155, http://dx.doi.org/10.1016/ j.physd.2008.10.006

[2] M. Golubitsky and I. Stewart, The Symmetry Perspective: From Equilibrium to Chaos in Phase Space and Physical Space., Birkhauser (2002)

[3] F. Hinkelman, D. Murrugarra, A. Jarrah, R. Laubenbacher, A Mathematical Framework for Agent Based Models of Complex Biological Networks, Bulletin of Mathematical Biology, 73(7) (2010), 1583-1602, http: //dx.doi.org/10.1007/S11538-010-9582-8

[4] E. Frias-Martinez, G. Williamson, V. Frias-Martinez, An Agent-Based Model of Epidemic Spread Using Human Mobility and Social Network Information, SocialCom/PASSAT 2011 Proceedings, Boston, MA, USA, 5764, http://dx.doi.org/10.1109/PASSAT/SocialCom.2011. 142

[5] B. Heath, R. Hill, F. Ciarallo, A Survey of Agent-Based Modeling Practices (January 1998 to July 2008), Journal of Artificial Societies and Social Simulation 12(4) (2009), 1-49, http://jasss.soc.surrey.ac.uk/12/4/9.html

[6] E. Bonabeau, Agent-Based Modeling: Methods and Techniques For Simulating Human Systems, PNAS, suppl. 3(99) (2002), 7280-7287, http://dx.doi.org/10.1073/pnas. 082080899

[7] S. Maerivoet, B. De Moor, Cellular Automata Models of Road Traffic, Physics Reports, 419(1) (2005), 1-64, http://dx.doi.org/10.1016/j.physrep.2005.08.005

[8] V. Grimm, S. F. Railsback Individual-based Modeling and Ecology, Princeton Series in Theoretical and Computational Biology (2005)

[9] S. Kaufmann, Metabolic Stability and Epigenesis in Randomly Constructed Genetic Nets, Journal of Theoretical Biology, 22(3) (1969), 437-467.

[10] F. Robert, Discrete Iterations: a Metric Study, SpringerVerlag (1986)

[11] I. Shmulevich, S. A. Kauffman, Activities and Sensitivities in Boolean Network Models, Physics Review Letters, 93(4) (2004), 048701 http://dx.doi.org/10.1103/ physrevlett.93.048701

[12] H. Mortveit, C. Reidys, An Introduction to Sequential Dynamical Systems, Springer (2008), http://dx.doi.org/10. 1007/978-0-387-49879-9 
[13] A. Veliz-Cuba, B. Aguilar, F. Hinkelmann, R. Laubenbacher, Steady State Analysis of Boolean Molecular Network Models via Model Reduction and Computational Algebra, BMC Bioinformatics, 15(1) (2014), 1-8, http: //dx.doi.org/10.1186/1471-2105-15-221

[14] R. Heckel, S. Schober, M. Bossert, Harmonic Analysis of Boolean Networks: Determinative Power and Perturbations, EURASIP Journal of Bioinformatics and System Biology 2013(6), (2013), 1-12, http://dx.doi.org/10.1186/ 1687-4153-2013-6

[15] R-S. Wang, A. Saadatpour and R. Albert, Boolean Modeling in Systems Biology: An Overview of Methodology and Applications, Physical Biology, 9(5) (2012), 055001, http://dx.doi.org/10.1088/1478-3975/9/5/055001

[16] A.T. Adai, S.V. Date, S. Wieland, E. M. Marcotte, LGL: Creating a Map of Protein Function with an Algorithm for Visualizing Very Large Biological Networks, Journal of Molecular Biology, 340 (2004), 179190, http://dx.doi. org/10.1016/j.jmb.2004.04.047

[17] A. Shamsul Arefin M. Inostroza-Ponta, L. Mathieson, R. Berretta, P. Moscato, Clustering Nodes in Large-Scale Biological Networks Using External Memory Algorithms, Proceedings of ICA3PP, Melbourne, Australia (2011), 375-386, http://dx.doi.org/10.1007/978-3-642-24669-2_ 36

[18] A. Veliz-Cuba, B. Stigler, Boolean Models Can Explain Bistability in the lac Operon, Journal of Computational Biology, 18(6) (2011), 783-794, http://dx.doi.org/ $10.1089 / \mathrm{cmb} .2011 .0031$

[19] A. Veliz-Cuba, B. Aguilar, R. Laubenbacher. Dimension Reduction of Large Sparse AND-NOT Network Models. Electronic Notes in Theoretical Computer Science, 316 (2015), 83-95, http://dx.doi.org/10.1016/j.entcs.2015.06. 012

[20] J. Guckenheimer, P. Holmes,Nonlinear Oscillations, Dynamical Systems, and Bifurcations of Vector Fields, Springer (2008)

[21] M. Oremland, R. Laubenbacher, Optimization of AgentBased Models: Scaling Methods and Heuristic Algorithms, Journal of Artificial Societies and Social Simulation, 17(2) (2014), 6, http://dx.doi.org/10.18564/jasss. 2472

[22] M. Oremland, R. Laubenbacher, Optimal Harvesting of a Predator-Prey Agent-Based Model Using Difference Equations. Bulletin of Mathematical Biology, 77(3) (2015), 434-459, http://dx.doi.org/10.1007/ s11538-014-0060-6
[23] S. Wolfram, Cellular Automata and Complexity: Collected Papers, Addison-Wesley (1994)

[24] J. M. Greenberg, S. P. Hastings, Spatial Patterns for Discrete Models of Diffusion in Excitable Media, SIAM Journal on Applied Mathematics, 34(3) (1978), 515-523, http://dx.doi.org/10.1137/0134040

[25] D. M. Wittmann, F. Bloechl, D. Truembach, W. Wurst, N. Prakash, F. J. Theis, Spatial Analysis of Expression Patterns Predicts Genetic Interactions at the MidHindbrain Boundary, PLOS Computational Biology, 11(5) (2009), 1-16, http://dx.doi.org/10.1371\%2Fjournal. pcbi.1000569

[26] J. E. Ferrell Jr., T. Yu-Chen Tsai, Q. Yang, Modeling the Cell Cycle: Why Do Certain Circuits Oscillate?, Cell, 144(6) (2011), 874-885, http://dx.doi.org/10.1016/j.cell. 2011.03.006

[27] R. Vabø, L Nøtterstad, An Individual Based Model of Fish School Reactions Predicting Antipredator Behaviour as Observed in Nature, Fisheries and Oceanography, 6(3) (1997), 155-171, http://dx.doi.org/10.1046/j.1365-2419. 1997.00037.x

[28] R.S. Olson, P. B. Haley, F. C. Dyer., C. Adami, Exploring the Evolution of a Trade-Off Between Vigilance and Foraging in Group-Living Organisms., Royal Society Open Science, (2015) 2:150135, http://dx.doi.org/10. 1098/rsos.150135

[29] R. Lukeman, Y. X. Li, L. Edelstein-Keshet, Inferring Individual Rules From Collective Behavior, Proceedings of the National Academy of Sciences, 107(28) (2010), 12576-12580, http://dx.doi.org/10.1073/ pnas.1001763107

[30] F. Gerlotto, S. Bertrand, N. Bez, M. Gutierrez, Waves of Agitation Inside Anchovy Schools Observed With Multibeam Sonar: a Way to Transmit Information in Response to Predation, ICES Journal of Marine Science, 63(8) (2006), 1405-1417, https://doi.org/10.1016/j. icesjms.2006.04.023

[31] B. Birnir, An ODE Model of the Motion of Pelagic Fish, Journal of Statistical Physics, 128(1) (2007), 535-568, https://doi.org/10.1007/s10955-007-9292-2

[32] Y. Y. Song, R. S. Zeng, J. F. Xu, J. Li, X. Shen, W. G. Yihdego, Interplant Communication of Tomato Plants through Underground Common Mycorrhizal Networks, PLOS One, 5(10) (2010), http://dx.doi.org/10. 1371/journal.pone.0013324 